\title{
Initial Study on Cancer-Related Cognitive Dysfunction With the Implementation of QEEG
}

\author{
Magda Żołubak ${ }^{1}$, Aleksandra Kawala-Janik ${ }^{1}$, Michał Podpora ${ }^{1}$, \\ Mariusz Pelc ${ }^{1,2}$, Wojciech Skowron ${ }^{3}$ \\ 1 Opole University of Technology, Faculty of Electrical Engineering, Automatic Control \\ and Informatics, Poland \\ 2 University of Greenwich, Department of Computing and Information Systems, Old \\ Royal Naval College, United Kingdom \\ ${ }^{3}$ Healthcare Center in Biała, Department of Internal Diseases, Biała, Poland
}

\begin{abstract}
Chemotherapy is one of the most common treatments used in cancer therapy despite its serious side effects, which remain a huge concern. To the most common side effects (apart from weight and hair loss) include memory and concentration problems as well as changes in taste. This paper presents a very preliminary study (with only one elderly female subject participating) focusing on cognitive dysfunction after chemotherapy in breast cancer. This phenomenon, called Cancer-Related Cognitive Dysfunction (CRCD), is a frequent occurrence. The obtained results prove the impact of chemotherapy on the participant's ability to concentrate. The QEEG method and digital data analysis were used as the measurement methods.
\end{abstract}

\section{Introduction}

This paper presents the impact of cancer treatment in the form of chemotherapy on impairment of cognitive and memory functions, and concentration deficits in elderly (postmenopausal) women suffering from breast cancer. The described phenomenon occurs very frequently and is called Cancer-Related Cognitive Dysfunction (CRCD) (Isenberg-Grzeda \& Ellis, 2017; Johns et al., 2016).

CRCD occurs in patients of all ages after chemotherapy, but it is most frequently observed in women suffering breast cancer. The level of dysfunction, however, is a very individual feature (Hermelink et al., 2017; Wefel et al., 2015).

The most common reason for the occurrence of CRCD may be the toxicity of certain ingredients of chemotherapeutic drugs. This also refers 
to substances that do not cross the brain-blood barrier or cause cytokine deregulation, despite the fact that the latter are being used in experimental treatment of some tumors (Jiang et al., 2017; Ren et al., 2017).

Other side effects of chemotherapy, apart from CRCD, are Taste Alterations (TA), fatigue, vomiting, nausea, and - most commonly - hair loss (Zabernigg et al., 2010).

The study presented in this paper is of a preliminary character. The performed tests proved the hypothesis concerning the occurrence of cognitive impairment after chemotherapy.

\section{Research Methodology}

For the purpose of this research (at a very preliminary stage), quantitative EEG (QEEG) (Blackburn et al., 2016) was applied and only one female subject was tested. The main aim of this study was to initially prove cognitive impairment in women in postmenopausal age who underwent chemotherapy for breast cancer. EEG was applied to the following locations: C3, C4, Fz, Cz, P3, P4, F3 and F4 - in accordance with the 1020 system (Homan et al., 1987).

The data was recorded in a frequency range of $1-70 \mathrm{~Hz}$ and notch filter (48-52 Hz - for Europe) was applied. The sampling frequency was $250 \mathrm{~Hz}$. The amplifier used for this study was a DigiTrack BF type digital electroencephalograph.

\section{QEEG - A Brief Introduction}

The authors decided to implement the QEEG for the purpose of this initial study due to the fact that the method in question has a very long tradition in research into concentration deficits (in, inter alia, children with ADHD), where application of the quantitative method gave very promising results (Ramirez et al., 2001), as well as in the analysis of motor action in adult persons (Azarpaikan \& Taheri Torbati, 2017). The basis of the quantitative method, similarly to the traditional method (EEG) is measurement of the electrical activity of the human brain. In the case of this type of research, supplementary information is obtained in addition to a record of the activity of brain waves. Owing to these special algorithms, it is possible to obtain, for instance, Fast Fourier Transform (FFT). Owing the more detailed information provided, the quantitative measurement method can form a basis for a more accurate diagnosis and enable to create a map 
of brain function, with better results when compared to traditional EEG (Żołubak, 2017).

\section{The Subject and the Performed Experiment}

The study is of a very preliminary character, its purpose being to prove the hypothesis, based on literature study, that chemotherapy can affect cognitive skills of elderly women suffering from cancer (Johns et al., 2016; van Dam et al., 1998; Zabernigg et al., 2010). The criteria for patient participation in the study specified the participants as those suffering from breast cancer with an already planned chemotherapy regimen. The participant of the initial stage of this research was an elderly female, aged 77, diagnosed with breast cancer, shortly after her first QEEG test, whose treatment had already begun. She underwent total unilateral mastectomy, with excision of regional lymph nodes. The chemotherapeutic agents she was administered were cytokines from the anthracycline group (the so-called "red chemotherapy").

After the fourth infusion the patient collapsed, as a result of which further infusions were discontinued. In the opinion of the lead doctor, this happened because the participant did not stop smoking cigarettes. Another QEEG study was conducted one year after the first test. The participant is currently on continuous hormonal therapy administered in the form of pills and is also taking medication for obstructive pulmonary disease. The patient's family reported that the subject's personality changed significantly after chemotherapy, describing her attitude as "malicious and unpleasant to others". During and after chemotherapy, the subject was taking various supplements, including B-group vitamins. The woman being a cigarette addict might have affected the results of both tests.

\section{Results and Discussion}

Based on the obtained EEG recordings, i.e. average values of amplitude within particular ranges (absolute values) and their percentages in the whole spectrum (relative values), together with average values of the amplitudes for the whole spectrum at each particular point, were analyzed.

The analysis was based on the control values for adults, in accordance with the tests carried out by Sterman (Sterman \& House, 1980; Tan et al., 2009). The first test before the cancer diagnosis was the control sample, whereas the second one measured the changes that occurred after chemotherapy. Figure 1 presents signals from electrodes C3 and C4 (40 sec- 

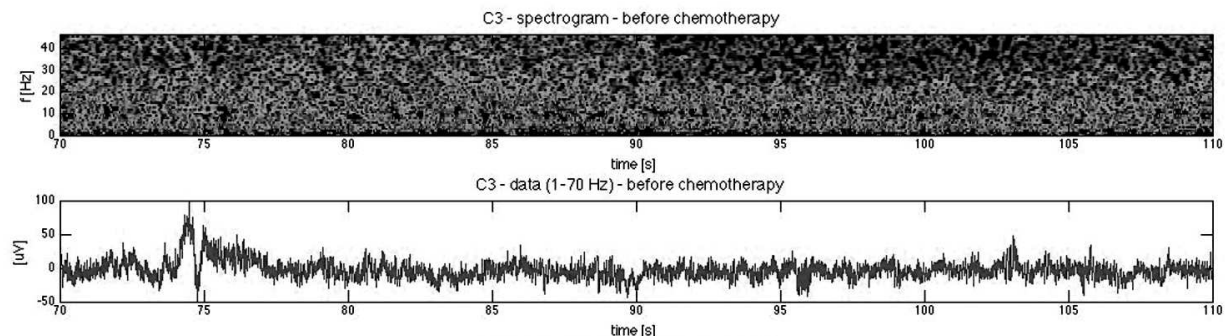

C4 - spectrogram - before chemotherapy
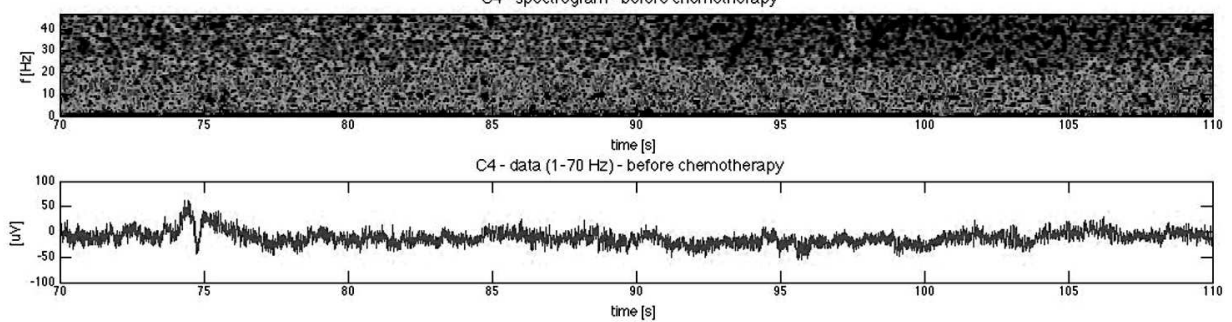

Figure 1. Spectrogram and EEG data from C3 and C4 electrodes, 40s of recording, eyes closed - before chemotherapy
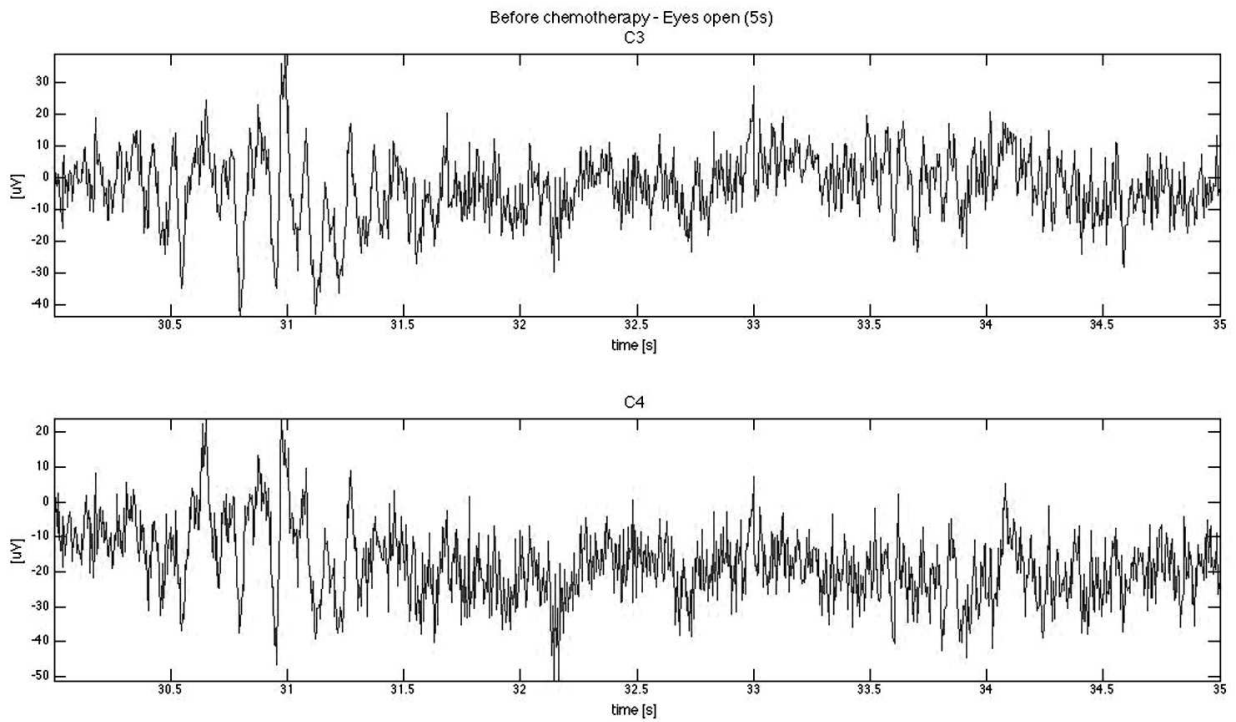

Figure 2. EEG data, eyes open, raw signal (1-70 Hz, Hardware-based processing), C3 (top) and C4 (bottom) - before chemotherapy

onds of data). The data was recorded at closed eyes before chemotherapy. Numerous artifacts are visible in the EEG spectrogram.

The EEG recordings show numerous artifacts such as eye blinking and eyes movement (Figure 2). 
After closing the eyes, the so-called "stop response" should occur, with a significant increase of Alpha frequency. However, this did not happen, possibly due to the participant's smoking habit (Domino et al., 2009). In Figure 3 , it is possible to observe (C3 electrode signal in particular) very slow (almost sleep) waves below $2 \mathrm{~Hz}$, instead of clean Alpha waves. The data was obtained before chemotherapy.
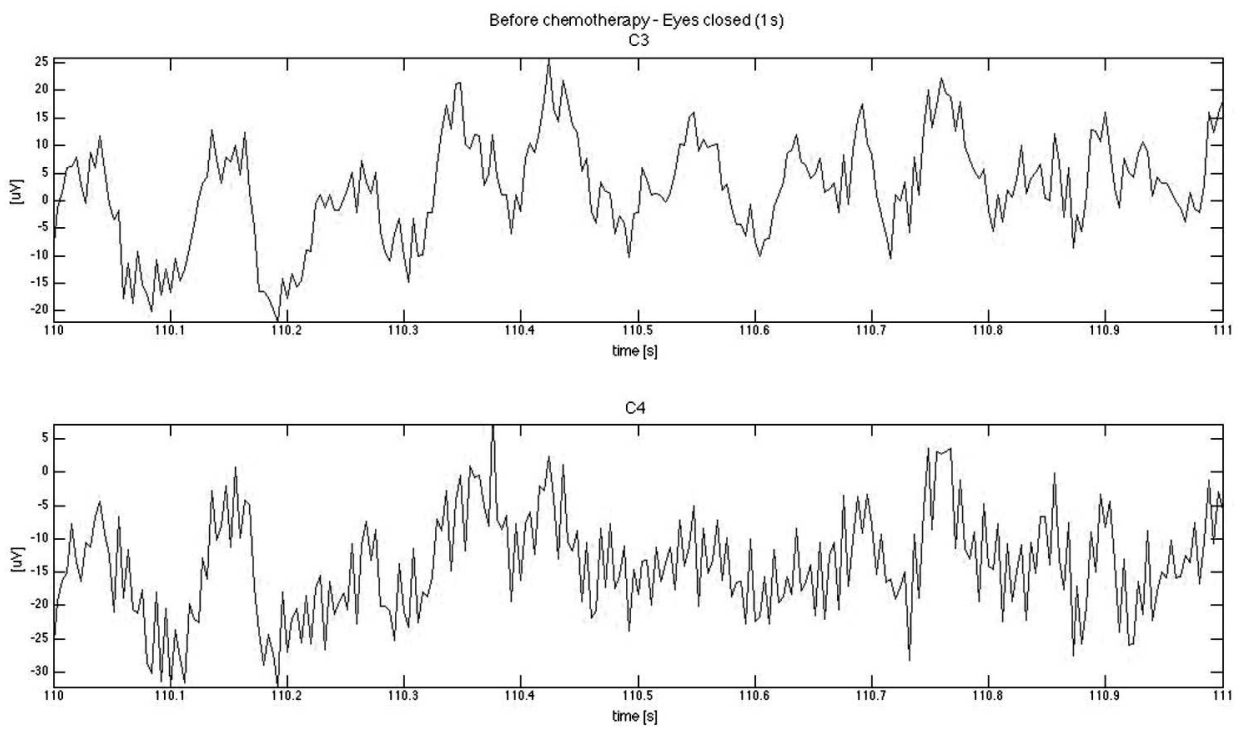

Figure 3. Eyes closed (1 random second), C3 (top) and C4 (bottom) - before chemotherapy, showing no "stop response"

It was possible to observe a decreased percentage of both Delta and Theta waves for the right hemisphere and the midline; for the right hemisphere, only Theta waves with increased amplitudes were observed.

The measurement points enabled to record above-norm amplitude values for the Alpha frequency, which may suggest short-term memory problems (Britton et al., 2016; Clarke et al., 2007; Markovska-Simoska \& PopJordanova, 2017). A higher Beta frequency amplitude, on the other hand, may indicate increased anxiety, as well as problems with concentration and sleep (Britton et al., 2016; Markovska-Simoska \& Pop-Jordanova, 2017).

After chemotherapy, EEG data still contained numerous artifacts and the administered treatment did not affect the signal in any significant way. The spectrogram shows numerous artifacts such as eye blinking and eye movement. However, an increase in Alpha frequency can be noticed after ca. 90 seconds, when the participant closed her eyes (see: close-ups in Figures 4 and 5). 

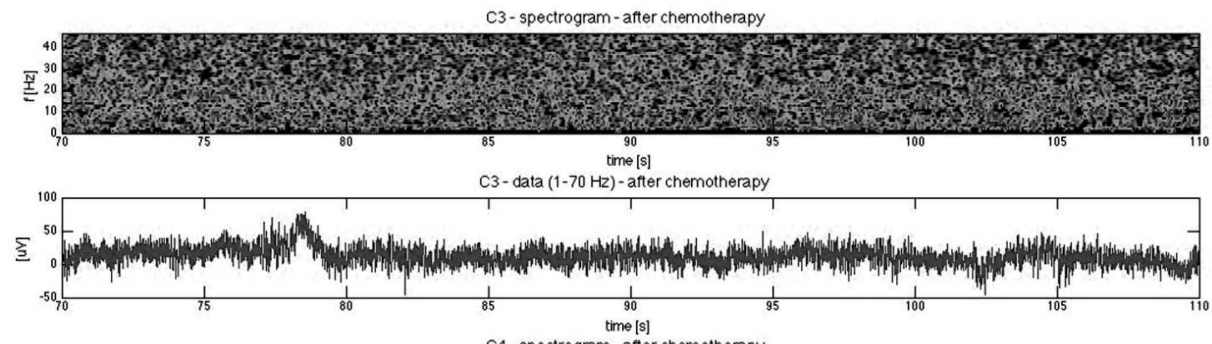

C4 - spectrogram - after chemotherapy

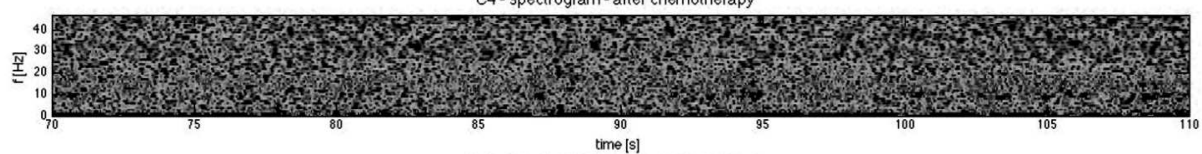

C4- data $(1-70 \mathrm{~Hz})$-after chemotherapy

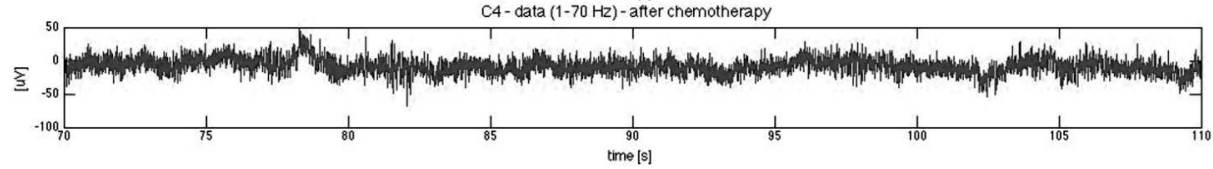

Figure 4. Spectrogram and EEG data from electrodes C3 and C4, 40s recording, eyes closed - after chemotherapy, with various artifacts such as eye blinking and eye movement
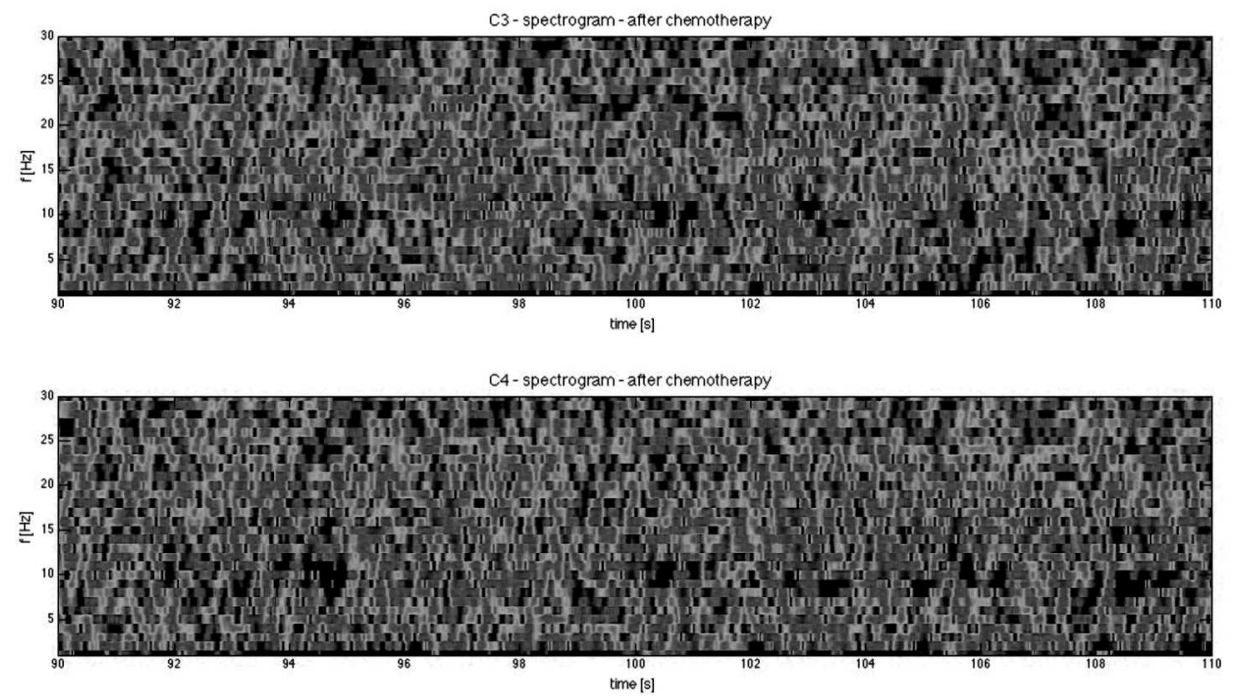

Figure 5. Spectrogram from electrodes C3 and C4, 30s recording, eyes closed, a close-up - after chemotherapy with various artifacts such as eye blinking and eye movement

It is still possible to notice very low frequency ranges (a spiky shape) similar to those present in the signal recorded before chemotherapy. Moreover, it is difficult to distinguish typical strong Alpha waves at closed eyes (Figure 6). 

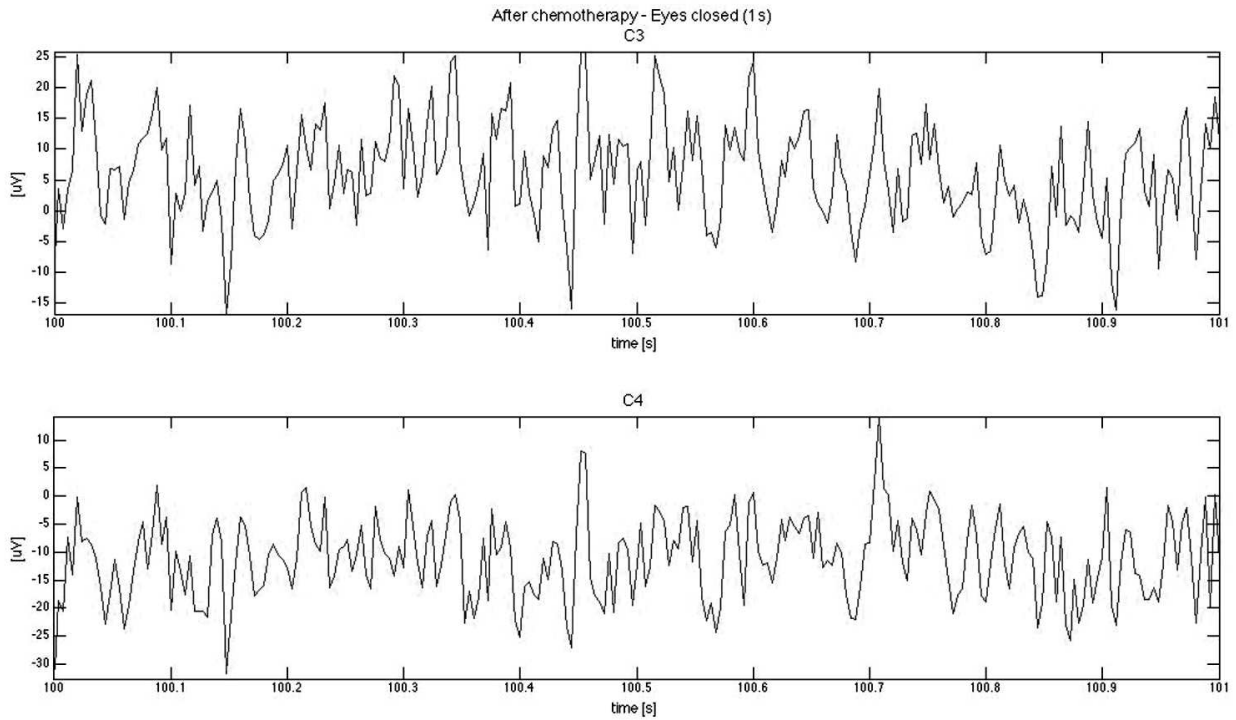

Figure 6. Eyes closed (1 random second), C3 (top) and C4 (bottom), typical strong Alpha waves absent

The signals still contained numerous artifacts, both before and after chemotherapy, but this is due to the nature of biomedical data. For open eyes, a decreased percentage of Delta, Theta, and Alpha waves can be noticed. The amplitude of Alpha frequencies increased in the areas of the left hemisphere and the midline whereas Beta frequencies are above norm. For closed eyes, Theta frequencies increased in the area of the left hemisphere. Both Alpha and Beta waves were above normal range.

After treatment, visible changes were recorded for Beta and Alpha waves, which correlate with the subject's attention and short-term memory, put her into chronic stress, caused anxiety and sleep problems. These adverse effects were noted in the patient's medical record and are not a result of the aging process. The changes were so pronounced that (based on literature review) cancer-related cognitive dysfunction may be suspected (Hermelink et al., 2017; Isenberg-Grzeda \& Ellis, 2017; Johns et al., 2016; Wefel et al., 2015).

The chosen method of measurement makes it possible to show the differences between the state before and after chemotherapy. With the QEEG, it was possible to detect changes for different frequency ranges, while quantitative EEG is an effective method for detecting even small changes present in cognitive disorders. The QEEG is also a fast and accurate method for evaluating cognitive changes after chemotherapy and can be used to diag- 
nose the extent of cognitive deficits. To confirm this, however, a larger group of patients needs to be studied.

\section{Further Research Plans}

Choosing the appropriate treatment for a specific patient is undoubtedly a difficult and responsible decision. This selection depends on numerous factors and despite the large number of available treatments, the great majority of cancer cases involve one of the following: surgery, chemotherapy, radiotherapy, or a combination of these. Advances in cancer treatment are undeniable, e.g. in the area of targeted (biological) therapies (Denduluri et al., 2016; Younes, 2011), yet these new approaches are very expensive in comparison with chemotherapy, which is the choice treatment in the majority of cases. For this reason, it is very important to mention the side effects and the impact of this type of treatment, without omission of the negative sides. Among these, negative effects, feelings or attitudes, which are often not objectively measurable (e.g. the quality of life, the feeling of humiliation, the feeling of loss of attractiveness, gloom/dejection), need to be emphasized (Von Minckwitz et al., 2017). By conducting the research study, the authors pinpoint the change that causes the patient to feel dispirited, believing that a deeper analysis of the influence of various types of treatment on the patient's life (e.g. by applying the QEEG to observe CRCD, as proposed in this paper) can result in lower prices and greater availability of other treatments and drugs.

It is necessary to follow up on the presented research, which will be performed by analyzing a greater number of patients, through the inclusion and comparison of various cases of breast cancer. It will also involve a deeper collaboration with clinicians, which will enable to obtain a more in-depth understanding of the changes that occur in patients during chemotherapy. This will be of a considerable help in developing improved data analysis methods and, as a result, may help to treat patients with CRCD.

It may also be necessary to adjust the impedance and calibration of the measuring equipment and to use different electrodes, such as gold cups or active electrodes.

To conclude, further plans include matching all parameters and selecting the most appropriate settings for the planned experiments on a larger group of subjects. 


\author{
R E F E R E N C E S
}

Azarpaikan, A., \& Taheri Torbati, H. (2017). Effect of somatosensory and neurofeedback training on balance in older healthy adults: a preliminary investigation. Aging Clinical and Experimental Research. doi: https://doi.org/10.1007/ s40520-017-0835-3

Blackburn, D., Yifan, Z., Bell, S., De Marco, M., He, F., Wilkinson, I., Farrow, T., et al. (2016). QEEG can distinguish patients with ad and volunteers. Journal of Neurology, Neurosurgery \& Psychiatry, 87(12). doi: http://dx.doi.org/10.1136/jnnp-2016-315106.56

Britton, J. W., Frey, L. C., Hopp, J. L., Korb, P., Lievens, W. E., Koubeissi, M. Z., Lievens, W. E., et al. (2016). Electroencephalography (EEG): An Introductory Text and Atlas of Normal and Abnormal Findings in Adults, Children, and Infants. Chicago: American Epilepsy Society.

Clarke, A. R., Barry, R. J., McCarthy, R., Selikowitz, M., Johnstone, S. J., Hsu, C. I., Magee, C. A., et al. (2007). Coherence in children with attentiondeficit/hyperactivity disorder and excess beta activity in their EEG. Clinical Neurophysiology, 118(7), 1472-1479.

Denduluri, N., Somerfield, M. R., \& Wolff, A. C. (2016). Selection of Optimal Adjuvant Chemotherapy Regimens for Early Breast Cancer and Adjuvant Targeted Therapy for HER2-Positive Breast Cancer: An American Society of Clinical Oncology Guideline Adaptation of the Cancer Care Ontario Clinical Practice Guideline Summary. Journal of Oncology Practice, 12(5), 485-488. doi: 10.1200/JOP.2016.012344

Domino, E. F., Ni, L., Thompson, M., Zhang, H., Shikata, H., Fukai, H., Sakaki, T., \& Ohya, I. (2009). Tobacco smoking produces widespread dominant brain wave alpha frequency increases. International Journal of Psychophysiology, 74(3), 192-198. doi: 10.1016/j.ijpsycho.2009.08.011

Hermelink, K., Bühner, M., Sckopke, P., Neufeld, F., Kaste, J., Voigt, V., Münzel, K., et al. (2017). Chemotherapy and Post-traumatic Stress in the Causation of Cognitive Dysfunction in Breast Cancer Patients. Journal of the National Cancer Institute, 109(10). doi: 10.1093/jnci/djx057

Homan, R., Herman, J., \& Purdy, P. (1987). Cerebral location of international 1020 system electrode placement. Electroencephalography and Clinical Neurophysiology, 66(4), 376-382.

Isenberg-Grzeda, E., \& Ellis, J. (2017). Cancer-related cognitive impairment. Current Opinion in Supportive and Palliative Care, 11(1), 17-18. doi: 10.1097/SPC.0000000000000256

Jiang, M., Zhang, W.-W., Liu, P., Yu, W., Liu, T., Yu, J. (2017). Dysregulation of SOCS-Mediated Negative Feedback of Cytokine Signaling in Carcinogenesis and Its Significance in Cancer Treatment. Frontiers in Immunology, 8:70. doi: 10.3389/fimmu.2017.00070

Johns, S. A., Von, Ah. D., Brown, L. F., Beck-Coon, K., Talib, T. L., Alyea, J. M., Monahan, P. O., et al. (2016). Randomized controlled pilot trial of mind- 
fulness-based stress reduction for breast and colorectal cancer survivors: effects on cancer-related cognitive impairment. Journal of Cancer Survivorship, 10(3), 437-448. doi: 10.1007/s11764-015-0494-3

Markovska-Simoska, S., \& Pop-Jordanova, N. (2017). Quantitative EEG in children and adults with attention deficit hyperactivity disorder: comparison of absolute and relative power spectra and theta/beta ratio. Clinical EEG and Neuroscience, 48(1), 20-32.

Ramirez, P. M., Desantis, D., \& Opler, L. A. (2001). EEG biofeedback treatment of ADD. A viable alternative to traditional medical intervention? Annals of the New York Academy of Sciences, 931, 342-358.

Ren, X., St. Clair, D. K., \& Butterfield, D. A. (2017). Dysregulation of cytokine mediated chemotherapy induced cognitive impairment. Pharmacological Research, 117, 267-273. doi: 10.1016/j.phrs.2017.01.001

Sterman, M. B., \& House, M. N. (1980). Quantitative analysis of training, sleep EEG and clinical response to EEG operant conditioning in epileptics. Electroencephalography and Clinical Neurophysiology, 49(5-6), 558-576.

Tan, G., Thornby, J., Hammond, D. C., Strehl, U., Canady, B., Arnemann, K., \& Kaiser, D. A. (2009). Meta-analysis of EEG biofeedback in treating epilepsy. Clinical EEG and Neuroscience, 40(3), 173-179.

van Dam, F. S., Schagen, S. B., Muller, M. J., Boogerd, W., vd Wall, E., Droogleever M. E., \& Rodenhuis, S. (1998). Impairment of cognitive function in women receiving adjuvant treatment for high-risk breast cancer: high-dose versus standard-dose chemotherapy. Journal of the National Cancer Institute, 90(3), 210-218.

Von Minckwitz, G., Procter, M. J., De Azambuja, E., Zardavas, D., Knott, A., \& Viale, G. (2017). APHINITY trial (BIG 4-11): A randomized comparison of chemotherapy $(\mathrm{C})$ plus trastuzumab $(\mathrm{T})$ plus placebo (Pla) versus chemotherapy plus trastuzumab $(\mathrm{T})$ plus pertuzumab $(\mathrm{P})$ as adjuvant therapy in patients (pts) with HER2-positive early breast cancer (EBC). Journal of Clinical Oncology, 35(18). doi: 10.1200/JCO.2017.35.18_suppl.LBA500

Wefel, J. S., Kesler, S. R., Noll, K. R., \& Schagen, S. B. (2015). Clinical characteristics, pathophysiology, and management of noncentral nervous system cancer-related cognitive impairment in adults. CA: A Cancer Journal for Clinicians, 65(2), 123-138. doi: 10.3322/caac.21258

Younes, A. (2011). Beyond chemotherapy: new agents for targeted treatment of lymphoma. Nature Reviews Clinical Oncology, 8(2), 85-96. doi: 10.1038/ nrclinonc.2010.189

Zabernigg, A., Gamper, E. M., Giesinger, J. M., Rumpold, G., Kemmler, G., Gattringer, K., Sperner-Unterweger, B., \& Holzner, B. (2010). Taste Alterations in Cancer Patients Receiving Chemotherapy: A Neglected Side Effect? The Oncologist, 15(8), 913-920. doi: 10.1634/theoncologist.2009-0333

Żołubak, M. (2017). The analysis method of a biological signal - QEEG. Zeszyty naukowe Politechniki Opolskiej, 75(365), 131-133. 